\title{
In vitro polyadenylation is stimulated by the presence of an upstream intron
}

\author{
Maho Niwa, Scott D. Rose, and Susan M. Berget \\ Marrs McClean Department of Biochemistry, Baylor College of Medicine, Houston, Texas 77030 USA
}

\begin{abstract}
The majority of vertebrate pre-mRNAs are both spliced and polyadenylated. To investigate the mechanism whereby processing factors recognize last exons containing both splicing and polyadenylation consensus elements, chimeric precursor RNAs containing a single intron and a poly(A) site were constructed and assayed for in vitro splicing and polyadenylation. Chimeric RNAs underwent splicing and polyadenylation. Both reactions occurred in a single RNA. The presence of an intron enhanced the rate of polyadenylation at a downstream poly(A) site. The extent of stimulation varied from two- to fivefold, depending on the magnesium concentration. Maximal stimulation of polyadenylation by an upstream intron required a $3^{\prime}$ splice site but not a $5^{\prime}$ splice site, suggesting that the structure of the terminal exon was more important than the presence of a complete upstream intron. We suggest that splicing and polyadenylation factors interact to recognize terminal, poly(A) site-containing exons. Such interaction may explain why all known intron-containing eukaryotic premRNAs generate their $3^{\prime}$ ends by polyadenylation.
\end{abstract}

[Key Words: pre-mRNA; polyadenylation; splicing]

Received June 1, 1990; revised version accepted July 13, 1990.

The production of mRNA in higher eukaryotes entails considerable RNA processing. Most vertebrate genes contain introns. Most, but not all, pre-mRNAs are polyadenylated. Although many intronless pre-mRNAs are polyadenylated, at least one major class, that coding for histone proteins, is not. In contrast, no mechanism other than polyadenylation for 3 '-end generation of spliced pre-mRNAs has ever been reported. This restriction hints at the existence of interaction between splicing and polyadenylation. $3^{\prime}$-Terminal exons begin with a $3^{\prime}$ splice site and terminate with a poly(A) site. They are longer, on average, than internal exons. A recent study of vertebrate exon size indicated an average length of 3'-terminal exons of 632 nucleotides versus a 137-nucleotide average for internal exons /Hawkins 1988). Some terminal exons are quite large. This difference also suggests that the processing machinery might recognize internal and $3^{\prime}$-terminal exons by different mechanisms.

Experiments investigating in vitro splicing and polyadenylation normally uncouple the two reactions. Poly(A) site-containing precursor RNAs lacking splicing signals polyadenylate, and vice versa. Furthermore, fractionation efforts indicate that the splicing and polyadenylation cleavage activities are distinct (Christofori and Keller 1988; Gilmartin et al. 1988; Takagaki et al. 1988). At first glance, these results would seem to contradict the possibility that splicing and polyadenylation communicate.

Mutation of either splicing or polyadenylation signals inhibits production of mature cytoplasmic RNA. Anal- ysis of the influence of one type of signal on the other processing reaction requires investigation of the nuclear phenotype of the mutation. Few experiments of this type have been reported. Those that have are suggestive of some link between the two processing steps. Villarreal and White (1983) reported that deletion of splicing signals depressed the level of polyadenylation of nuclear RNA at the downstream poly(A) site, resulting in normal levels of nuclear RNA with dispersed 3' termini. Furthermore, placing an intron upstream, but not downstream, of a poly(A) site increases expression from transfected genes (Buchman and Berg 1988) and raises the level of nuclear poly $(\mathrm{A})^{+}$RNA (Huang and Gorman 1990), suggesting that maximal polyadenylation and transport to the cytoplasm require both the presence of splicing signals and the correct positioning of these signals upstream of the poly(A) site.

Several experiments have indicated that vertebrate poly(A) sites work only within the appropriate context. Placing a functional polyadenylation cassette in the middle of an intron results in no apparent usage of the poly(A) site and normal levels of splicing, suggesting that poly(A) sites cannot be recognized when located between 5' and 3' splice sites (Adami and Nevins 1988; Brady and Wold 1988; Levitt et al. 1989). The same cassette placed within an exon, however, directs efficient polyadenylation. When placed in an intron, mutation of the flanking splicing signals permits recognition of the poly(A) site (Adami and Nevins 1988; Levitt et al. 1989). These results suggest that either splicing occurs so quickly that polyadenylation cannot compete or that 
there is a required polarity for the arrangement of processing signals in a pre-mRNA for both splicing and polyadenylation.

We chose to address the question of interaction between these two steps in processing by constructing chimeric splicing/polyadenylation precursor RNAs and assaying their activity in in vitro systems competent for both reactions. We find that the presence of an intron increases the rate of polyadenylation at a downstream poly(A) site. Maximal activity was dependent on the presence of a 3 ' splice site but not a $5^{\prime}$ splice site, suggesting that poly(A) sites are recognized as parts of terminal exons.

\section{Results}

To examine the interaction of polyadenylation and splicing factors during pre-mRNA processing, we constructed precursor RNAs that contain both splicing and polyadenylation signals (Fig. 1). The chimeric DNA constructed for this study (MXSVL) consists of 217 nucleotides from the major late transcription unit of human adenovirus containing two exons and a single intron (MX) fused within exon 2 to a standard polyadenylation cassette of 237 nucleotides from the late transcription unit of SV40 (SVL). Precursor RNAs made from only the splicing or polyadenylation portion of the chimeric gene splice or polyadenylate well, respectively, in in vitro HeLa cell extracts (Sperry and Berget 1986; Zillmann et al. 1987). MX DNA contains no known polyadenylation signals, and the SVL cassette contains no splicing signals. Fusion creates a DNA that transcribes into a 416-nucleotide precursor RNA with a second exon of 237 nucleotides, with a cleavage and poly(A) addition site located 182 nucleotides downstream of the $3^{\prime}$ splice site.

Processing reactions were performed in HeLa nuclear extracts capable of both splicing and polyadenylation under conditions that represented a compromise between standard splicing and polyadenylation assays $(1.5$ mM $\mathrm{MgCl}_{2}$; see Materials and methods). Reactions contained cordycepin $\left(3^{\prime} \mathrm{dATP}\right)$ to permit addition of only a single A residue following cleavage. The chimeric MXSVL substrate underwent both splicing and polyadenylation (Fig. 2). Final spliced and polyadenylation product RNA (referred to as $\mathrm{S}^{+} \mathrm{A}^{+} \mathrm{RNA}$ ) appeared by 20 min of reaction. Two other nonlariat RNAs, corresponding in molecular weight to polyadenylated, but not spliced, RNA $\left(\mathrm{A}^{+} \mathrm{S}^{-} \mathrm{RNA}\right)$ and spliced, but not polyadenylated, RNA ( $\left.\mathrm{S}^{+} \mathrm{A}^{-} \mathrm{RNA}\right)$, were also observed. The reaction products designated as polyadenylated $\left(\mathrm{A}^{+}\right)$in Figure 2 disappeared when reactions were performed in the presence of ATP instead of cordycepin; instead, processed material appeared above precursor RNA, indicating the addition of a poly(A) tail and confirming the identification of the $\mathrm{A}^{+}$species. $\mathrm{S}^{+} \mathrm{A}^{-}$RNA was present in equal amounts in reactions performed in the presence of cordycepin or ATP, indicating that this species was not polyadenylated. Mapping of the polyadenylated product RNA with complementary riboprobes indicated
A
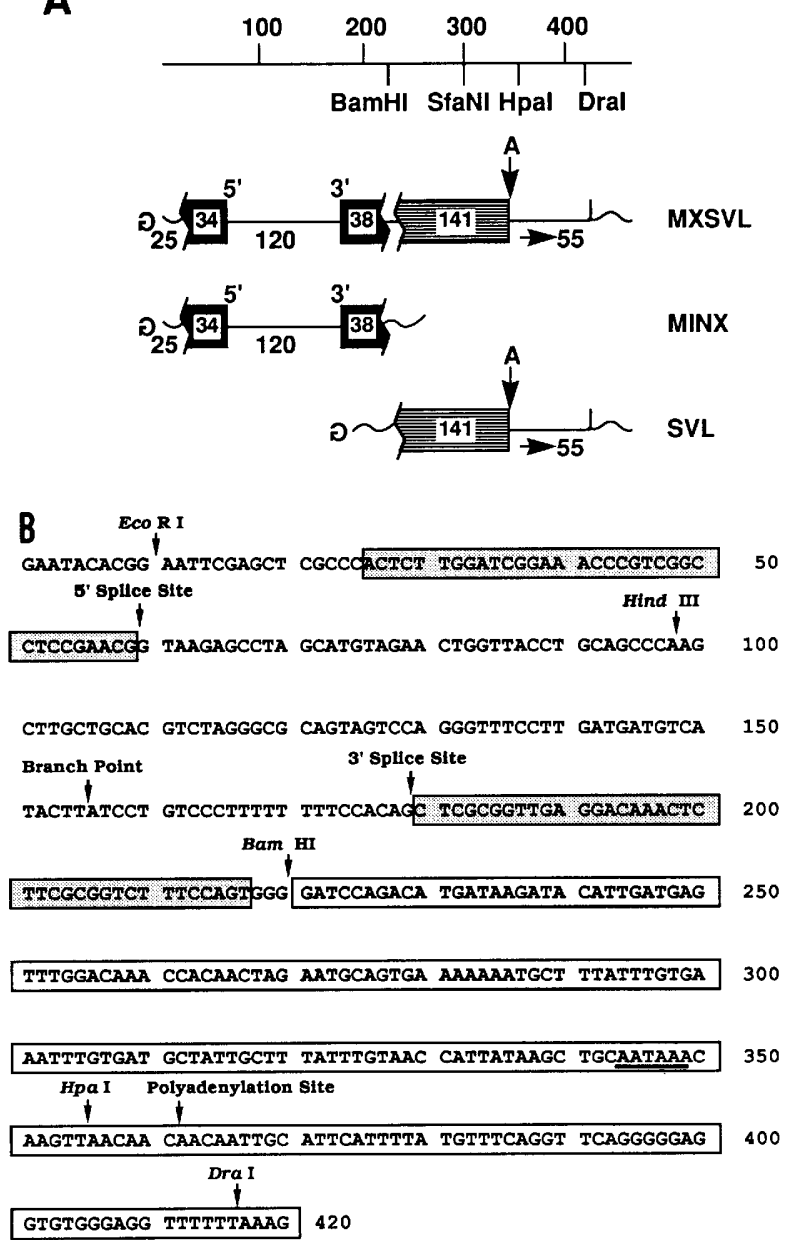

Figure 1. Chimeric splicing/polyadenylation precursor RNAs. (A) Large boxes indicate exon sequences; lines represent introns and sequences downstream of the poly(A) addition site; wavy lines indicate vector sequences. Solid and lined boxes represent exon sequences from the adenovirus 2 major late and SV40 late transcription units, respectively. Splice sites $\left(5^{\prime}\right.$ and $\left.3^{\prime}\right)$, poly(A) addition sites, and restriction enzyme sites used to truncate in vitro transcription templates are indicated. $(B)$ Sequence of MXSVL chimeric precursor RNA. Shaded boxes demarcate exon sequences from the adenovirus major late transcription unit; open boxes indicate sequences from the SV40 late transcription unit. MXSVL5' was a deletion of this sequence, removing the sequences from the indicated EcoRI-HindIII sites. MXSVL3' was a point mutant in which the $3^{\prime}$ splice site was altered from an AG to an AA.

that the site of cleavage was the correct in vivo-utilized site (data not shown).

Production of $\mathrm{S}^{+} \mathrm{A}^{+}$RNA species indicated that both splicing and polyadenylation reactions occurred on the same precursor molecule. $\mathrm{A}^{+} \mathrm{S}^{-}$RNA consistently appeared before $\mathrm{S}^{+} \mathrm{A}^{+}$RNA. More complete time courses detected $\mathrm{A}^{+} \mathrm{S}^{-}$RNA as early as $3 \mathrm{~min}$ into the reaction (data not shown, but see Fig. 3D), indicating that the chimeric substrates were extremely active for polyadenylation. $\mathrm{S}^{+} \mathrm{A}^{-}$RNA and $\mathrm{S}^{+} \mathrm{A}^{+}$RNA, however, were not 

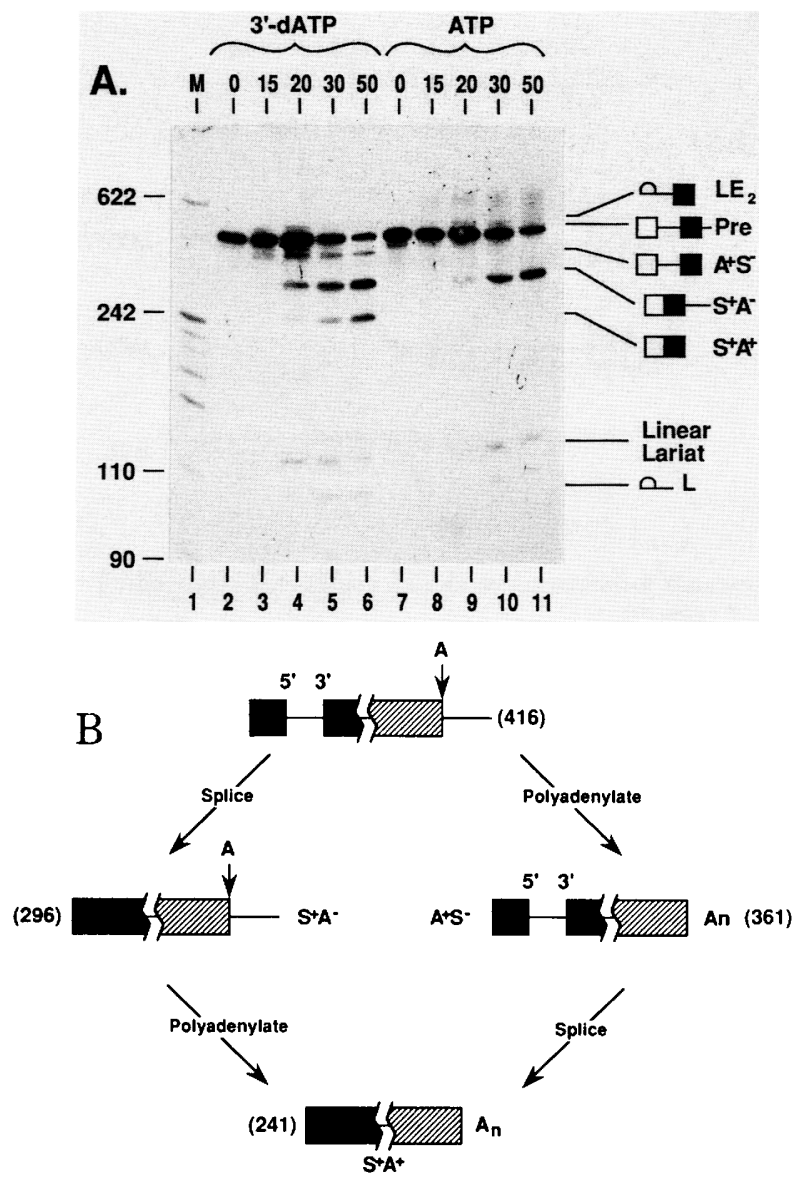

Figure 2. Time course of splicing and polyadenylation of chimeric precursor RNAs. In vitro processing reactions of MXSVL performed in the presence of $3^{\prime}$ dATP or ATP were sampled for RNA on a $5 \%$ denaturing acrylamide gel. RNA species are as described in $B$. Exon 2 is shaded. Polyadenylated species appear as a smear above precursor RNA in the + ATP lanes. Those bands present in lanes 2-6, but missing from lanes $7-11$, are RNAs that have undergone polyadenylation cleavage and the addition of only one A residue in the presence of cordycepin and poly(A) addition in the presence of ATP. Species were identified as indicated in Materials and methods. Marker bands are HpaII fragments of pBR322. (B) Pathway of splicing and polyadenylation for chimeric RNAs. (Pre) Precursor $\mathrm{RNA}\left(\mathrm{A}^{+} \mathrm{S}^{-}\right)$ polyadenylated, nonspliced $\mathrm{RNA}\left(\mathrm{S}^{+} \mathrm{A}^{-}\right)$spliced, nonpolyadenylated RNA; $\left(\mathrm{S}^{+} \mathrm{A}^{+}\right)$spliced and polyadenylated RNA are abbreviations used to identify RNA species in all figures. The lengths of MXSVL precursor, intermediate, and product RNAs are indicated.

visible until $15 \mathrm{~min}$ of incubation, suggesting that polyadenylation is the preferred first processing reaction.

The presence of a 3', but not $5^{\prime}$, splice site is required for maximal polyadenylation in chimeric RNAs

To ask whether the splicing signals present in MXSVL RNA affected polyadenylation, mutant precursor RNAs lacking splicing signals were created. MXSVL3' con- tained a point mutation (AG to AA) at the $3^{\prime}$ splice site beginning at exon 2. MXSVL5 was deleted for exon 1 and its $5^{\prime}$ splice site. When incubated in in vitro processing extracts, MXSVL3' precursor RNA produced no spliced product or released lariat, reflecting the effect of a mutated 3' splice site on splicing (Fig. 3A). A small amount of lariat-exon 2 was generated. A similar partial splicing phenotype has been observed previously for 3 ' splice site point mutations by use of RNAs that contain only splicing signals (Aebi et al. 1986; Lamond et al. 1987).

Mutation of the 3' splice site affected the efficiency of polyadenylation at the end of the exon. Less total polyadenylated RNA was observed at all time points of reaction with mutant than with wild-type RNA. For the mutant RNA, only one polyadenylated product RNA species was created. For wild-type RNA, two polyadenylated species were created: RNA that had not been spliced $\left(\mathrm{A}^{+} \mathrm{S}^{-} \mathrm{RNA}\right)$ and RNA that had been spliced $\left(A^{+} S^{+}\right.$RNA). Thus, to compare polyadenylation efficiencies, the amount of $\mathrm{A}^{+} \mathrm{S}^{-}$RNA for the mutant must be compared to the total amount of $\mathrm{A}^{+} \mathrm{S}^{-}$and $\mathrm{S}^{+} \mathrm{A}^{+}$ RNA for wild-type precursor RNA. To assess the total effect of mutation of the $3^{\prime}$ splice site, extended time courses were performed, and the amount of product RNA was determined by scanning the RNA gel in a Betascope 603 blot analyzer (see Materials and methods). A plot of femtomoles of polyadenylated product RNA versus time of incubation for both mutant and wild-type RNAs is shown in Figure 3D. Wild-type precursor RNA underwent polyadenylation at a rate 2.1 -fold better than did the mutant (determined from the slopes of the curves in Fig. 3D). Thus, a single base change at the $3^{\prime}$ splice site depressed polyadenylation at a downstream poly $(\mathrm{A})$ site, indicating that maximal polyadenylation with chimeric precursor RNAs was dependent on the presence of wild-type upstream splicing signals.

In contrast, deletion of exon 1 and its 5 ' splice site had little effect on the levels of polyadenylation (Fig. 3C and D). The amount of polyadenylated RNA from the mutant was equivalent to the sum of the amounts of $\mathrm{A}^{+} \mathrm{S}^{-}$ and $\mathrm{S}^{+} \mathrm{A}^{+}$RNAs for wild-type precursor RNA. Deletion of exon 1 produced an RNA precursor with an isolated terminal exon beginning with all known splicing signals at the $3^{\prime}$ end of the intron and terminating with a poly(A) site. Therefore, when a terminal exon was intact, polyadenylation efficiency of chimeric precursor RNAs was maximal. The phenotypes of these two mutants suggested that it was not the presence of an intron per se that activated polyadenylation cleavage at a downstream site but the presence of the poly $(\mathrm{A})$ site within a valid exon (and therefore downstream of a $3^{\prime}$ splice site). This property suggests that poly(A) sites are recognized as parts of terminal exons.

\section{Chimeric RNAs polyadenylate at a higher rate than isolated poly $(A)$ sites}

The chimeric MXSVL RNA contains all of the SV40 late sequences normally present in a standard SV40 late precursor RNA used to study polyadenylation in a reaction 

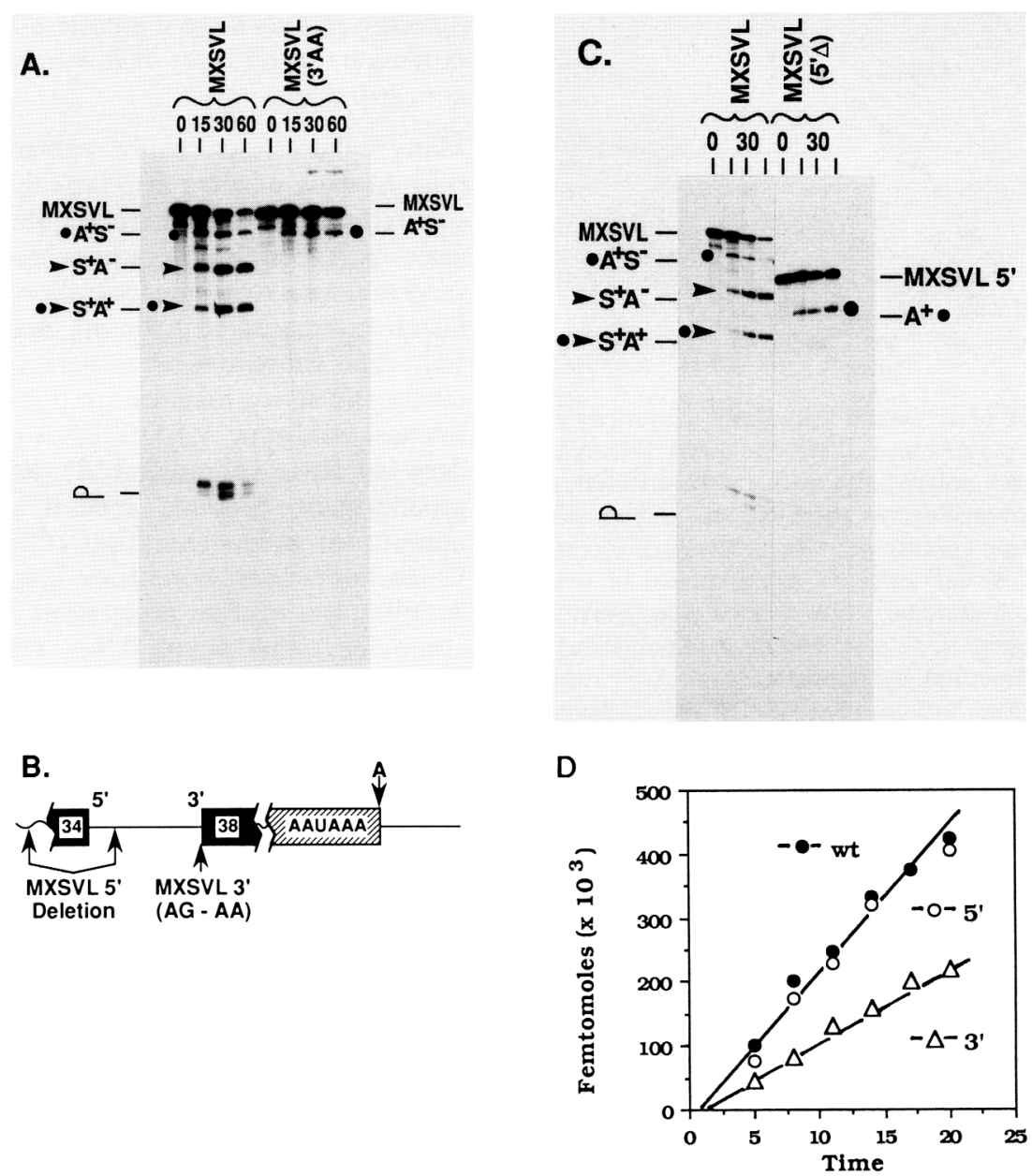

Figure 3. The 3', but not the 5', splice site is required for maximal polyadenylation of chimeric precursor RNAs. Mutant and wild-type chimeric substrates (diagrammed in $B)$ were incubated in splicing/polyadenylation reactions for the indicated times. $(A$ and C) Polyacrylamide gels of wild-type and mutant RNA reactions. Spliced RNAs $\left(\mathrm{S}^{+} \mathrm{A}^{-}\right.$and $\left.\mathrm{S}^{+} \mathrm{A}^{+}\right)$are indicated with an arrowhead; polyadenylated RNAs $\left(\mathrm{A}^{+}, \mathrm{A}^{+} \mathrm{S}^{-}\right.$and $\left.\mathrm{S}^{+} \mathrm{A}^{+}\right)$are indicated with a solid circle. (D) Quantitation of reaction. Equal amounts (fmoles) of wild-type and mutant RNAs were processed in vitro. Reactions were sampled at more time points than in the gels in $A$ or $C$. Production of polyadenylated RNA from mutants $\left(\mathrm{A}^{+}\right.$species from $A$ and $\left.C\right)$ and wild-type $\left(\mathrm{A}^{+} \mathrm{S}^{-}\right.$and $\mathrm{S}^{+} \mathrm{A}^{+}$from $A$ and $\left.C\right)$ was quantitated and displayed with respect to time of reaction. Rates were calculated from slopes.

uncoupled from splicing. This SV40 late poly(A) site is one of the most active poly(A) sites studied in vitro. We compared the polyadenylation activity of the chimeric MXSVL RNA to that of isolated SVL RNA under the same conditions (Fig. 4). The rate of polyadenylation of isolated SV40 RNA was lower than that of the chimeric RNA; quantitation by Beta-scan indicated a 2.4-fold increase in the initial rate of polyadenylation of the chimeric RNA compared to that of the isolated poly(A) site, indicating that the environment of the poly $(\mathrm{A})$ site in the chimeric RNA precursor altered the ability of extract factors to polyadenylate this site. Presumably, it is the presence of the upstream intron that is facilitating the polyadenylation reaction.

It should be noted that the polyadenylation rate being compared was that occurring at very early times of the reaction before significant splicing occurred (i.e., the appearance of $\mathrm{A}^{+} \mathrm{S}^{-}$RNA). Thus, it is not the splicing reac- tion per se that accelerated polyadenylation but events earlier than activity, presumably assembly of splicing factors with precursor RNA, that facilitated reaction. The first splicing complexes form within $1 \mathrm{~min}$ of incubation in vitro. The early acceleration of polyadenylation in the chimera suggested that these complexes and their associated factors were responsible for boosting polyadenylation in vitro.

Table 1 compares the efficiency of polyadenylation in a set of experiments with wild-type and mutant RNAs in which the amount of introduced precursor RNA varied from 100 to 3000 fmoles. Our extracts were capable of processing up to 3000 fmoles of input RNA without saturating either the polyadenylation or splicing activity (data not shown), although the actual rate of polyadenylation varied from extract to extract. Table 1 represents experiments yielding rates of polyadenylation varying from 4 to $27 \mathrm{fmoles} / \mathrm{min}$ that were 


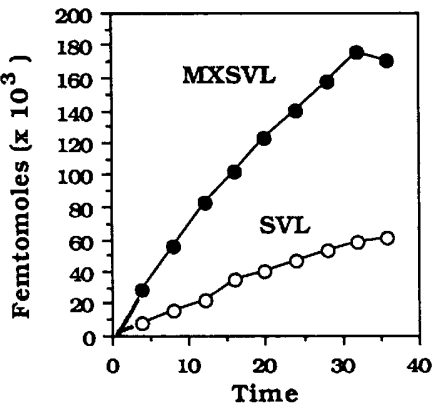

Figure 4. The presence of an intron stimulates polyadenylation at a downstream poly(A) site. Chimeric RNA (MXSVL) containing the SV40 late poly(A) site and a polyadenylation precursor RNA (SVL) containing just the SV40 late poly(A) site were prepared to the same specific activity and assayed for polyadenylation in vitro. Equal amounts (femtomoles) of precursor were compared. Individual RNA species were quantitated by scanning polyacrylamide gels. The rates of polyadenylation of MXSVL (represented by femtomoles of $\mathrm{A}^{+} \mathrm{S}^{-}$and $\mathrm{S}^{+} \mathrm{A}^{+} \mathrm{RNAs}$ ) and SVL (femtomoles of $\mathrm{A}^{+} \mathrm{RNA}$ ) are displayed with respect to time of reaction.

performed with different extracts over a 1-year period of time. In each case, despite different rates of reaction, alteration of the $3^{\prime}$ splice site reduced the rate of polyadenylation two- to threefold, and alteration of the $5^{\prime}$ splice site had little effect. Thus, the effects we are observing appear to be a property of the substrate and not the extract. Furthermore, the effect of mutation of the 3' splice site was identical to removal of all splicing signals, supporting the idea that the sequences boosting polyadenylation in chimeric precursor RNAs were splicing signals.

Table 1. Effect of mutation of splicing signals on polyadenylation of chimeric MXSVL RNA

\begin{tabular}{|c|c|c|c|}
\hline Precursor & $\begin{array}{l}\text { Polyadenylation } \\
\text { rate } \\
\text { (fmole/min) }\end{array}$ & & $\begin{array}{l}\text { lative rate } \\
t / \text { mutant })\end{array}$ \\
\hline MXSVL & 11.3 & & 2.7 \\
\hline MXSVL 3' & 4.1 & & \\
\hline MXSVL & 3.8 & & 2.2 \\
\hline MXSVL $3^{\prime}$ & 1.7 & & \\
\hline MXSVL & 6.0 & & 2.5 \\
\hline MXSVL $3^{\prime}$ & 2.4 & & \\
\hline MXSVL & 27.3 & & 2.1 \\
\hline \multirow[t]{2}{*}{ MXSVL $3^{\prime}$} & 13.0 & & \\
\hline & & average & e 2.4 \\
\hline MXSVL & 27.3 & & 1.0 \\
\hline MXSVL 5' & 27.3 & & \\
\hline MXSVL & 4.3 & & 1.3 \\
\hline \multirow[t]{2}{*}{ MXSVL 5' } & 3.4 & & \\
\hline & & average & e 1.1 \\
\hline MXSVL & 3.8 & & 1.9 \\
\hline SVL & 2.0 & & \\
\hline MXSVL & 6.0 & & 3.0 \\
\hline \multirow[t]{2}{*}{ SVL } & 2.0 & & \\
\hline & & average & 2.4 \\
\hline
\end{tabular}

Splicing signals have a greater effect on polyadenylation rates at low magnesium concentrations

Many laboratories study polyadenylation of isolated poly(A) sites in vitro at relatively low magnesium concentrations. To see whether splicing signals would still affect polyadenylation rates when reactions were examined at magnesium concentrations that favor polyadenylation compared to splicing, reactions of mutant and wild-type substrates were performed in reactions containing no added magnesium (Fig. 5A). Under these conditions, wild-type MXSVL RNA polyadenylated but underwent little splicing. $\mathrm{A}^{+} \mathrm{S}^{-}$RNA was therefore the predominant product species. Quantitation of the amount of polyadenylation of wild-type and mutant RNAs under these conditions (Fig. 5B) indicated that the 3 ' splice site mutant underwent polyadenylation poorly compared to wild-type RNA. Under these conditions, the difference in rate of polyadenylation between wildtype and mutant RNAs was fivefold. Thus, splicing signals have a greater effect on polyadenylation rates at lower magnesium concentrations. Removal of the first exon in the $5^{\prime}$ deletion mutant continued to have minimal effects on polyadenylation at lowered magnesium concentrations. We interpret these results to indicate
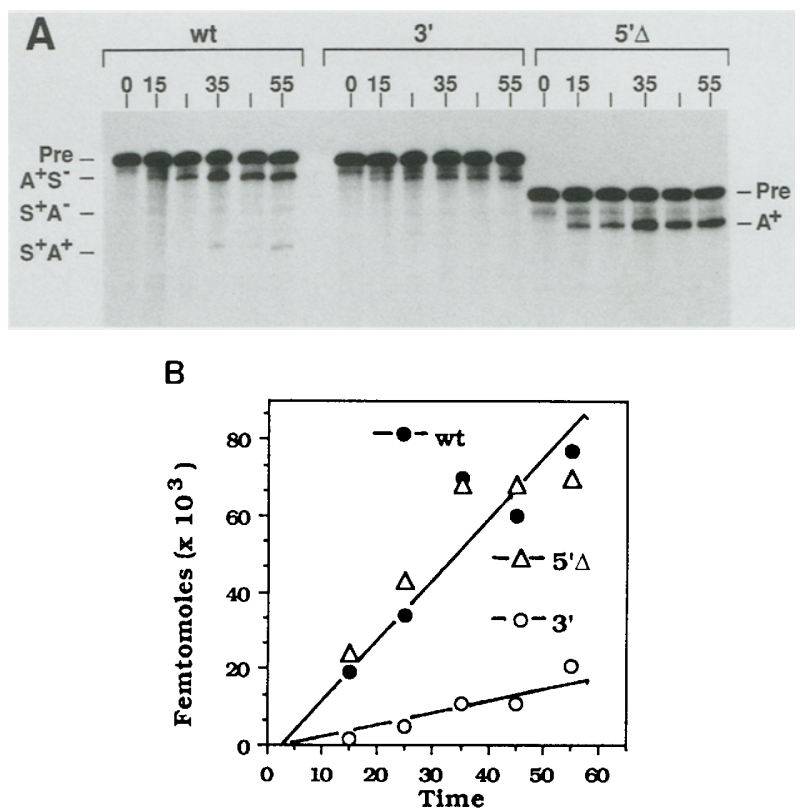

Figure 5. Splicing signals affect polyadenylation rates greater at low magnesium concentrations. Wild-type MXSVL, MXSVL3', and MXSVL5' were incubated in processing extract in the absence of added magnesium. Because nuclear extract was dialyzed against EDTA during the final step of preparation, the endogenous magnesium in these reactions is estimated to be very low, $<0.2 \mathrm{~mm}$. Samples were removed and displayed on denaturing acrylamide gels. (A) Polyadenylated, but not spliced, $\mathrm{A}^{+} \mathrm{S}^{-}$product RNAs are indicated for wild type. The amount of product in $A$ was quantitated by gel scanning. $(B)$ Rates of reaction were calculated from the slopes of the lines. 
that the interaction between polyadenylation and splicing signals causing a boost to polyadenylation in the chimeric RNAs operates best at lower magnesium concentrations.

\section{Discussion}

Most, but not all, vertebrate pre-mRNAs contain introns and form their $3^{\prime}$ termini by polyadenylation. Historically, the relationship between these two processing steps has been confusing and controversial. Early in vivo experiments indicated that polyadenylation generally precedes splicing (Nevins and Darnell 1978), although several reports have detected removal of introns distal to the poly(A) site before polyadenylation (Berget and Sharp 1978; Beyer and Ohshiem 1988). The possibility remained, however, that removal of the last intron of a pre-mRNA was linked to polyadenylation in some way.

In this communication, we discuss an examination of the relationship between splicing and polyadenylation by use of an in vitro system competent for both reactions and a simple chimeric precursor RNA containing two exons in which the second exon terminated with a poly(A) site. We found that this chimeric RNA spliced and polyadenylated at high efficiency, with both reactions occurring in a single RNA. The first intermediate visible in the reaction was that creating a polyadenylated, but not spliced, RNA. This intermediate was observed as early as $3 \mathrm{~min}$ of reaction. This reaction preference was observed in many experiments conducted over a 2-year period of time with a variety of extract preparations that varied in their total polyadenylation activity. These properties suggest an ordered pathway for reaction for a terminal exon in which polyadenylation precedes splicing.

The presence of an upstream intron activated polyadenylation at a downstream poly(A) site. Mutation of splicing signals reduced polyadenylation levels to that of the isolated poly(A) site, suggesting that the boost in activity was caused by the presence of the intron. Activation was dependent on the $3^{\prime}$ splice site but not on exon 1 or its $5^{\prime}$ splice site. These results suggest that stimulation of polyadenylation results not from the presence of an upstream intron, but rather from the presence of a valid last exon beginning with splicing signals at the $3^{\prime}$ end of the intron (including the branchpoint, pyrimidine track, and $3^{\prime}$ splice site) and ending with polyadenylation signals.

The boost to polyadenylation that we observed occurred within minutes of the start of incubation, at a time considerably before splicing could be detected. Furthermore, stimulation was observed at low magnesium concentrations $(<0.5 \mathrm{~mm})$ under which little actual splicing activity occurred. These results suggest that it is the assembly of splicing factors with precursor RNA that is responsible for the boost in polyadenylation activity, rather than the splicing reaction itself. The first splicing complex on this intron forms within $1 \mathrm{~min}$ of incubation (Zillmann et al. 1988). It also forms in the absence of magnesium; later complexes do not form under these conditions (data not shown). Thus, we antic- ipate that the splicing factors involved in formation of the first large splicing complex are stimulating polyadenylation.

One other in vitro study of chimeric RNAs has appeared (Ryner and Manley 1987) in which the investigators concluded that in vitro splicing and polyadenylation were occurring independently on natural SV40 early precursor RNA. They observed that cordycepin inhibited polyadenylation but not splicing, suggesting that the two reactions are uncoupled. We cannot address these results directly because we find efficient polyadenylation of SV40 late and of chimeric RNAs containing SV40 late sequences in the presence of cordycepin. Presumably, this difference reflects reaction and extract differences. We agree that splicing and polyadenylation activity can be uncoupled in vitro. Presumably, this uncoupling was responsible for the residual polyadenylation activity in our mutant constructs with impaired splicing signals. Our experiments suggest that although the splicing and polyadenylation catalytic machineries may be different entities, these machineries interact to orchestrate the correct recognition of terminal exons and introns.

The precursor RNA used in this study combines a $5^{\prime}$ proximal intron from the adenovirus major late transcription unit fused to the last several hundred bases of the SV40 late transcription unit. These two sequences splice and polyadenylate very efficiently when assayed in vitro as isolated sites (Sheets et al. 1987; Zarkower and Wickens 1987, 1988; Zillmann et al. 1987, 1988; Ryner et al. 1987). In fact, these are probably the two most active in vitro precursor RNAs yet described. We chose these signals because they are the two about which the most is known with respect to consensus sequences and interacting factors. However, by this choice, we have asked one strong signal to influence the activity of the other. We are presently constructing chimeric RNAs with weaker signals to see if the enhancement increases. We are also investigating splicing and polyadenylation of valid last introns and exons. The constructs in this communication did not include either intron or exon signals proximal to a $3^{\prime}$ splice site terminating a valid last intron. It is possible that such natural constructs will have different properties than those reported here.

Our data best fit a model in which polyadenylation precedes splicing. A variety of experiments have been performed suggesting the reverse (Adami and Nevins 1988; Brady and Wold 1988; Levitt et al. 1989). In these experiments poly(A) sites (without splicing signals) naturally reside or have been placed within introns, and the effect of such introduction on splicing of the resident intron has been measured. In all of these cases, no polyadenylation occurred and normal splicing occurred. Incapacitation of the splicing signals activated polyadenylation. This result has usually been interpreted to indicate that splicing "beats" polyadenylation, for example, that assembly of splicing factors and commitment to splicing precedes commitment to polyadenylation. A perspective of interaction between splicing and polyadenylation 
suggests an alternative explanation of these data. All of these constructions create an aberrant polar arrangement of processing signals. Last exons begin with a $3^{\prime}$ splice site and terminate with a poly(A) site. When a poly(A) site is placed within an intron, the order of sites becomes $3^{\prime}$ splice site, $5^{\prime}$ splice site, and poly(A) site. If there is interaction between splicing and polyadenylation, presumably it occurs between the factors that bind to sequences at the $3^{\prime}$ end of the intron and those that bind poly $(\mathrm{A})$ sites. The presence of an intervening $5^{\prime}$ splice site would be predicted to interfere with this communication, and mutation of the $5^{\prime}$ splice site would be predicted to restore it. Thus, we would suggest that placement of a poly(A) site within an intron inactivates the poly(A) site, not because the polyadenylation machinery cannot compete with the splicing machinery in this context but because it cannot interact with it.

Most vertebrate genes contain introns and are polyadenylated. To our knowledge, no eukaryotic pre-mRNA that contains introns generates its $3^{\prime}$ termini by any means other than polyadenylation, suggesting that the terminal exon in a split gene can only be recognized if it is terminated by a poly(A) site. Schizosaccharomyces pombe U6 RNA, however, contains a pre-mRNA-like intron and is not polyadenylated (Tani and Oshima 1990 ), indicating that intron removal is not obligatorily coupled to polyadenylation in noncoding precursor RNAs. Marzluff (Pandey and Marzluff 1987; Pandey et al. 1990) has reported that intron-containing histone genes are inhibited for $3^{\prime}$-end formation by the normal U7-mediated $3^{\prime}$-end generating mechanism used by unsplit histone genes. Instead, they rely on polyadenylation, activating downstream cryptic sites. This result suggests that the presence of an intron favors 3 '-end formation by polyadenylation.

\section{Materials and methods}

\section{Precursor RNAs}

The MXSVL chimeric RNA was constructed by fusion of 217 nucleotides from the MINX precursor RNA to 237 nucleotides from the SV40 late poly(A) site at the BamHI site in the SP64 polylinker, terminating the MINX construct and the natural BamHI site from the SV40 late region (Zillmann et al. 1987). Three nucleotides of polylinker separate the first half of exon 2 from the adenovirus major late region in MINX from the beginning of the SV40 late region. The SV40 sequences continued 96 nucleotides past the poly $(\mathrm{A})$ addition site, at which point it is fused to the SP64 polylinker via a $B a m / B c l$ linkage. This precursor is diagrammed in Figure 1. The 3' splice site mutation of adenovirus intron 1 was provided by R. Padgett (Wang and Padgett 1989). A HindIII-ScaI fragment was used to replace wildtype intronic sequences of MXSVL to create MXSVL $3^{\prime}$. MXSVL $5^{\prime}$ was created by deletion of all MXSVL exon 1 sequences /from an EcoRI site within the SP6 polylinker at nucleotide 10 of MXSVL to a HindIII site located within the intron). Mutations were sequenced before use.

\section{Splicing reactions}

Unless otherwise indicated, splicing/polyadenylation reactions contained $1 \mathrm{~mm}$ creatine phosphate, $0.25 \mathrm{~mm}$ ATP, $1.0 \mathrm{~mm} \mathrm{3}$
dATP, $1.5 \mathrm{mM} \mathrm{MgCl}_{2}, 0.37 \mathrm{~mm}$ dithiothreitol, $0.9 \%$ polyethylene glycol, $44 \mathrm{~mm} \mathrm{KCl}, 8.8 \mathrm{mM}$ Tris- $\mathrm{Cl}(\mathrm{pH} 7.9\}, 8.8 \%$ glycerol, $0.2 \mathrm{~mm}$ EDTA, and $44 \%$ nuclear extract. RNAs were extracted after reaction and analyzed as described previously. In vitro splicing and polyadenylation were routinely performed using the same nuclear extract but under slightly different conditions. Splicing assays contained higher magnesium concentrations (1.5-2.5 mM vs. $0-1.0 \mathrm{mM}$ ) and salt than polyadenylation reactions. These investigations used a set of conditions representing a compromise between the two maxima $1.5 \mathrm{mM}$ $\mathrm{MgCl}_{2} ; 44 \mathrm{mM} \mathrm{KCl}$ ) in which both polyadenylation and splicing occurred. In addition, to facilitate observation of cleavage products without interfering with poly(A) addition, ATP was replaced with 3' dATP (cordycepin). In the presence of cordycepin, polyadenylation cleavage occurs, and poly(A) addition is terminated after the addition of a single A residue (Jacob and Rose 1983; Sheets et al. 1987). We refer to molecules that have undergone single A addition as being polyadenylated. Control reactions indicated that replacement of ATP with $3^{\prime}$ dATP had no effect on splicing efficiency in templates lacking poly(A) sites (data not shown).

A variety of intermediate RNAs were produced from the reaction of chimeric templates. Linear intermediate and product species were identified by molecular weight and the disappearance of those products that had undergone poly $(\mathrm{A})$ addition under conditions in which ATP replaced cordycepin $\left(\mathrm{A}^{+} \mathrm{S}^{-}\right.$and $\mathrm{A}^{+} \mathrm{S}^{+}$species). Lariat species were identified by their alternative migration in gels with different percentages of acrylamide and their comigration with species produced in splicing reactions of MINX RNA.

\section{Quantitation of processing reaction}

Polyacrylamide gels of RNA products from processing reactions were quantitated by scanning in a Betagen Betascope 603 blot analyzer. The amount of product produced was calculated from a knowledge of the specific activity of the [32]UTP-labeled precursor RNA, U content of each RNA species, and the determined efficiency of counting in the instrument $(15 \%)$. When wild-type and mutant substrates were compared, substrates were carefully prepared to the same specific activity and reacted by using the same reaction/extract mix. Polyadenylation and splicing rates were identical over at least a 20 -fold concentration of precursor RNA, indicating that reactions were performed under nonsaturating conditions. Precursor RNAs both react and are degraded in processing extracts. Analysis of total counts for each substrate during reaction indicated that each of the precursors used in this study (MXSVL, MXSVL3', MXSVL5', and SVL) had a similar half-life in extract. Rates were calculated from early time points in the reaction before product decay contributed significantly to disappearance of total counts.

\section{Acknowledgments}

This work was supported by grants from the American Cancer Society (NP-695) and Texas Advanced Technologies Program to S.M.B. We thank Dr. Ed Murphey and the M.D. Anderson Tumor Biology Department for access to the Betagen 603 scanner, Gilbert Cote for data analysis, and Becky Moore and Dixie Brewer for technical assistance.

The publication costs of this article were defrayed in part by payment of page charges. This article must therefore be hereby marked "advertisement" in accordance with 18 USC section 1734 solely to indicate this fact. 


\section{References}

Adami, G. and J.R. Nevins. 1988. Splice site selection dominates over poly(A) site choice in RNA production from complex adenovirus transcription units. EMBO J. 7: 2107-2116.

Aebi, M., H. Hornig, R.A. Padgett, and C. Weissmann. 1986. Sequence requirements for splicing of higher eucaryotic nuclear pre-mRNA. Cell 47: 555-565.

Berget, S.M. and P.A. Sharp. 1978. Structure of late adenovirus 2 heterogeneous nuclear RNA. J. Mol. Biol. 129: 547-559.

Beyer, A. and Y.N. Ohsheim. 1988. Splice site selection, rate of splicing, and alternative splicing on nascent transcripts. Genes Dev. 2: 754-765.

Brady, H.A. and W.S.M. Wold. 1988. Competition between splicing and polyadenylation reactions determines which adenovirus E3 mRNAs are synthesized. Mol. Cell. Biol. 8: $3291-3297$.

Buchman, A. and P. Berg. 1988. Comparison of intron-dependent and intron-independent gene expression. Mol. Cell. Biol. 8: 4395-4405.

Christofori, G. and W. Keller. 1988. 3' cleavage and polyadenylation of mRNA precursors in vitro requires a poly(A) polymerase, a cleavage factor, and a snRNP. Cell 54: 875-889.

Gilmartin, G.M., M.A. McDevitt, and J.R. Nevins. 1988. Multiple factors are required for specific RNA cleavage at a poly(A) addition site. Genes Dev. 2: 578-587.

Hawkins, J.D. 1988. A survey on intron and exons lengths. $\mathrm{Nu}$ cleic Acids Res. 16: 9893-9908.

Huang, M.T.F. and C.M. Gorman. 1990. Intervening sequences increase efficiency of RNA 3' processing and accumulation of cytoplasmic RNA. Nucleic Acids Res. 18: 937-947.

Jacob, S.T. and K.M. Rose. 1983. Poly|A/ polymerase from eucaryotes. In Enzymes of nucleic acid synthesis and modification (ed. S.T. Jacob), vol. 2, pp. 35-157. CRC Press, Boca Raton.

Lamond, A.I., M. Konarska, and P.A. Sharp. 1987. A mutational analysis of spliceosome assembly: Evidence for splice site collaboration during spliceosome formation. Genes Dev. 1: $532-543$

Levitt, N., D. Briggs, A. Gil, and N.J. Proudfoot. 1989. Definition of an efficient synthetic poly(A) site. Genes Dev. 3: $1019-1025$.

Nevins, J.R. and J.E. Darnell, Jr. 1978. Steps in the processing of Ad2 mRNA: Poly $(A)^{+}$sequences are conserved and poly(A) addition precedes splicing. Cell 15: 1477-1487.

Pandey, N.B. and W.F. Marzluff. 1987. The stem-loop structure at the $3^{\prime}$ end of histone mRNA is necessary and sufficient for regulation of histone mRNA stability. Mol. Cell. Biol. 7: 4557-4559.

Pandey, N.B., N. Chodchoy, T.J. Liu, and W.F. Marzluff. 1990. Introns in histone genes alter the distribution of $3^{\prime}$ ends. Nucleic Acids Res. 18: 3161-3170.

Ryner, L.C. and J.L. Manley. 1987. Requirement for accurate and efficient mRNA 3 ' end cleavage and polyadenylation of an SV40 pre RNA in vitro. Mol. Cell. Biol. 7: 495-503.

Sheets, M.D., P. Stephenson, and M.P. Wickens. 1987. Products of in vitro cleavage and polyadenylation of simian virus 40 late pre-mRNAs. Mol. Cell. Biol. 7: 1518-1529.

Sperry, A.O. and S.M. Berget. 1986. In vitro cleavage of the simian virus 40 early polyadenylation site adjacent to a required downstream TG sequence. Mol. Cell. Biol. 6: 47344741 .

Takagaki, Y., L.C. Ryner, and J.L. Manley. 1988. Separation and characterization of a poly(A) polymerase and a cleavage/ specificity factor required for pre-mRNA polyadenylation. Cell 52: 731-742.
Tani, T. and Y. Oshima. 1990. The gene for U6 small nuclear RNA in fission yeast has an intron. Nature 337: 87-90.

Villarreal, L.P. and R.T. White. 1983. A splice junction deletion deficient in the transport of RNA does not polyadenylate nuclear RNA. Mol. Cell. Biol. 3: 1381-1388.

Wang, X. and R.A. Padgett. 1989. Hydroxyradical "footprinting" of RNA: Application to pre-mRNA splicing complex. Proc. Natl. Acad. Sci. 86: 7795-7799.

Zarkower, D. and M. Wickens. 1987. Specific pre-cleavage and post-cleavage complexes involved in the formation of SV40 late mRNA 3' termini in vitro. EMBO I. 6: 4185-4192.

. 1988. A functionally redundant downstream sequence in SV40 late pre-mRNA is required for mRNA $3^{\prime}$ end formation and for assembly of a pre-cleavage complex. $I$. Biol. Chem. 263: 5780-5788.

Zillmann, M., S.D. Rose, and S.M. Berget. 1987. U1 small nuclear ribonucleoproteins are required early during spliceosome assembly. Mol. Cell. Biol. 7: 2877-2883.

Zillmann, M., M.L. Zapp, and S.M. Berget. 1988. Gel electrophoretic isolation of splicing complexes containing small nuclear ribonucleoprotein particles. Mol. Cell Biol. 8: 814821 . 


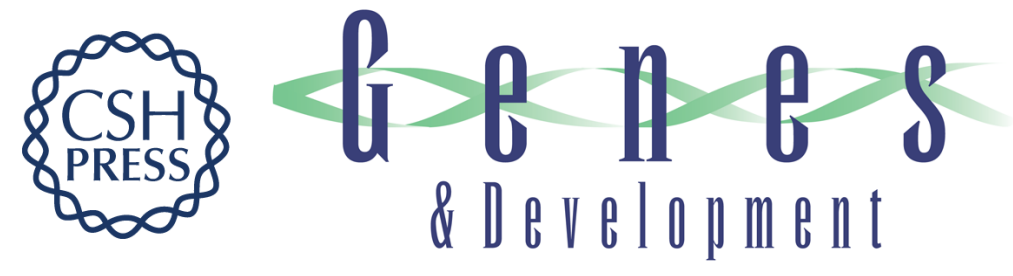

\section{In vitro polyadenylation is stimulated by the presence of an upstream intron.}

M Niwa, S D Rose and S M Berget

Genes Dev. 1990, 4:

Access the most recent version at doi:10.1101/gad.4.9.1552

References This article cites 26 articles, 15 of which can be accessed free at:

http://genesdev.cshlp.org/content/4/9/1552.full.html\#ref-list-1

License

Email Alerting Receive free email alerts when new articles cite this article - sign up in the box at the top Service right corner of the article or click here.

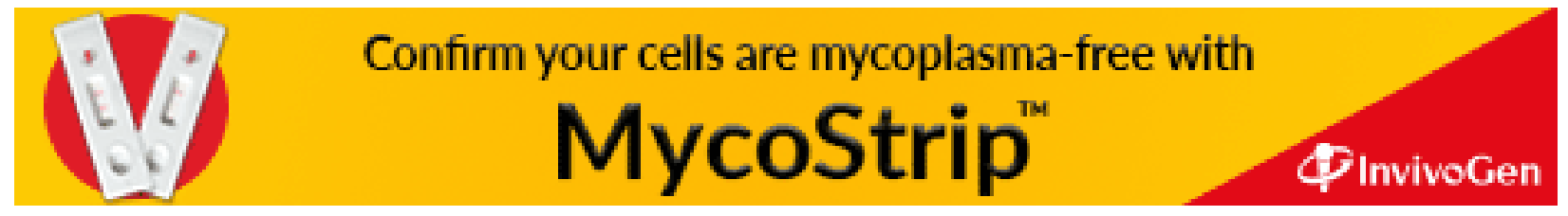

